\title{
A method for rapid generation of 3D infrared radiation intensity field of engine exhaust plume
}

Bao, Xing-dong, Yu, Xi-long, Wang, Zen-hua, Mao, Hongxia, Xiao, Zhi-he

Xing-dong Bao, Xi-long Yu, Zen-hua Wang, Hong-xia Mao, Zhi-he Xiao, "A method for rapid generation of 3D infrared radiation intensity field of engine exhaust plume," Proc. SPIE 11455, Sixth Symposium on Novel Optoelectronic Detection Technology and Applications, 114555F (17 April 2020); doi: $10.1117 / 12.2565064$

Event: Sixth Symposium on Novel Photoelectronic Detection Technology and Application, 2019, Beijing, China 


\title{
A Method for Rapid Generation of 3D Infrared Radiation Intensity
}

\section{Field of Engine Exhaust Plume}

\author{
BAO Xing-dong ${ }^{1,2,3}$, YU Xi-long ${ }^{1,2}$, WANG Zhen-hua ${ }^{3}$, MAO Hong-xia ${ }^{3}$ XIAO Zhi-he ${ }^{3}$
}

\author{
(1. State Key Laboratory of High Temperature Gas Dynamics, Institute of Mechanics, Chinese \\ Academy of Sciences, Beijing 100190, China; \\ 2. School of Engineering Science, University of Chinese Academy of Sciences, Beijing 100049, \\ China; \\ 3. Science and Technology on Optical Radiation Laboratory, Beijing 100854, China)
}

\begin{abstract}
Rocket engine exhaust plume produces a strong infrared radiation signal and is widely used for target diagnosis and detection. The traditional method for calculating the infrared radiation characteristics of the exhaust plume is difficult and time-consuming. In this paper, the engineering analytical method in the band is used to calculate the equivalent spectral radiation intensity of the exhaust plume, and the spectral radiation intensity varies with the viewing angle. The relationship curve constructs the spectral radiance intensity as a function of the viewing angle. This method does not just take advantage of the efficiency of the engineering analysis method, but also preserves the accuracy of numerical simulation. The spatial distribution of the infrared radiation intensity field in the typical band of the exhaust plume is simulated by an example.
\end{abstract}

Keywords: exhaust plume; Infrared spectroscopy; three-dimensional space; radiation intensity; calculation method

\section{INTRODUCTION}

Exhaust plume has the characteristics of high temperature, high speed and multi-species flow. The radiation mechanism is very complex in the plume flow field. The gases of the high temperature and high pressure in the plume flow field which is several times of its own size. Exhaust plume infrared signals are important basis of diagnosing, detecting and identifying plume spectrum. ${ }^{[1 \sim 3]}$ Recent years, more and more attention has been paid to the research of flow and radiation characteristics of complex plume, and many useful insights have been gained. Scholars from various countries spend a lot of time studying the fine simulation model of tail flame flow and radiation characteristics. The computational fluid dynamics method ${ }^{[4-5]}$ is used to calculate the flow characteristics of the exhaust plume. The finite volume method ${ }^{[6]}$, Monte Carlo method ${ }^{[7]}$ and six-flow method ${ }^{[8 \sim 9]}$ are used to calculate the radiation characteristics of exhaust plume. The above method can obtain fine flow field structure and spectral characteristics, but it takes a lot of calculation time. This time cost is enormous when calculating the characteristics of the exhaust plume radiation for such a change in the viewing angle with a small angular interval. In this paper, it is found that the flow field structure of the exhaust plume at each typical height is similar, that is, a cylindrical envelope with a high-temperature radial transition to a low temperature in the axial direction, and its projected area is affected by the viewing angle. Since the physical form of the exhaust plume and the position of the emission band of the relevant components are fixed, based on the above knowledge, the spectral analysis intensity of the equivalent black body of the exhaust plume is calculated and fitted to the spectrum. The relationship between the radiation intensity and the change of the viewing angle, so as to achieve a fast

Sixth Symposium on Novel Optoelectronic Detection Technology and Applications, edited by Junhao Chu, Huilin Jiang Proc. of SPIE Vol. 11455, 114555F · ( 2020 SPIE · CCC code: 0277-786X/20/\$21 · doi: 10.1117/12.2565064 
estimation of the infrared spectrum radiation intensity of the full-space exhaust plume.

\section{ARITHMETIC MODEL}

The emission gas of the exhaust plume in the infrared band mainly includes $\mathrm{H}_{2} \mathrm{O}, \mathrm{CO}_{2}$ and $\mathrm{CO}$. Establish a spectral absorption coefficient characterization function for each component gas, such as the spectral absorption coefficient, and establish a spectral line average spacing characterization function for each component gas, such as the average line spacing. Based on the spectral line average spacing characterization function of each component gas, the spectral line overlap parameters are established. Calculate the average line spacing of $\mathrm{H}_{2} \mathrm{O}$, the average line spacing of $\mathrm{CO}_{2}$, and the average line spacing of $\mathrm{CO}$ using the following formula:

$$
\begin{aligned}
& \beta_{H 2 O}\left(n_{\text {wavenumber }}, T\right)=2 \pi \gamma_{H 2 O}\left(n_{\text {wavenumber }}, T\right) / d_{H 2 O}\left(n_{\text {wavenumber }}, T\right) \\
& \beta_{C O 2}\left(n_{\text {wavenumber }}, T\right)=2 \pi \gamma_{C O 2}\left(n_{\text {wavenumber }}, T\right) / d_{C O 2}\left(n_{\text {wavenumber }}, T\right) \\
& \beta_{C O}\left(n_{\text {wavenumber }}, T\right)=2 \pi \gamma_{C O}\left(n_{\text {wavenumber }}, T\right) / d_{C O}\left(n_{\text {wavenumber }}, T\right)
\end{aligned}
$$

Here, $n_{\text {wavenumber }}$ represents the corresponding wave number, $T$ is the temperature of the gas, $\gamma_{H 2 O}\left(n_{\text {wavenumber }}, T\right)$ is the average half width of the line of $\mathrm{H}_{2} \mathrm{O}, \gamma_{\mathrm{CO}_{2}}\left(n_{\text {wavenumber }}, T\right)$ is the average half width of the line of $\mathrm{CO}_{2}$, and $\gamma_{C O}\left(n_{\text {wavenumber }}, T\right)$ is the average half width of the line of CO.

In this paper, the average half width of the line is taken as the Doppler half width, then:

$$
\begin{aligned}
& \gamma_{\mathrm{H} 2 \mathrm{O}}\left(n_{\text {wavenumber }}, T\right)=\left[2 k T(\operatorname{In} 2) / m_{\mathrm{H} 2 \mathrm{O}} c^{2}\right]^{1 / 2} n_{\text {wavenumber }} \\
& \gamma_{\mathrm{CO} 2}\left(n_{\text {wavenumber }}, T\right)=\left[2 k T(\operatorname{In} 2) / m_{\mathrm{CO} 2} c^{2}\right]^{1 / 2} n_{\text {wavenumber }} \\
& \gamma_{\mathrm{CO}}\left(n_{\text {wavenumber }}, T\right)=\left[2 k T(\operatorname{In} 2) / m_{\mathrm{CO}} c^{2}\right]^{1 / 2} n_{\text {wavenumber }}
\end{aligned}
$$

Here, $k$ is the Boltzmann constant, $m_{\mathrm{H} 2 \mathrm{O}} 、 m_{\mathrm{CO} 2} 、 m_{\mathrm{CO}}$ represents the molar mass of the $\mathrm{H}_{2} \mathrm{O}$, the molar mass of the $\mathrm{CO}_{2}$ and the molar mass of the $\mathrm{CO}$, and $c$ is the speed of light.

Take an initial temperature $T_{i n i}$ and substitute to obtain the spectral radiance value corresponding to the wave number $n_{\text {wavenumber }}$ :

$$
\begin{aligned}
& I\left(n_{\text {wavenumber }}, T_{\text {ini }}\right)=\sigma T_{\text {ini }}{ }^{4}\left(2 R_{\text {plume }}\right) L_{\text {plume }}\left(1-e^{-W / d}\right) / \pi
\end{aligned}
$$

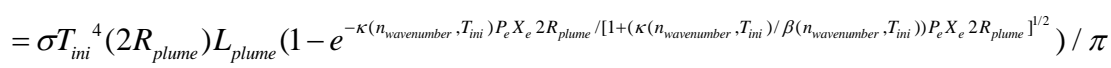

Here, $R_{\text {plume }}$ represents the plume diffusion radius, $L_{\text {plume }}$ represents the plume diffusion length, $\kappa\left(n_{\text {wavenumber }}, T_{\text {ini }}\right)$ is the absorption coefficient of the above gas, and $\beta\left(n_{\text {wavenumber }}, T_{i n i}\right)$ is the average line spacing of the above gases. Since the temperature change calculated by the above formula is close to linear, iteratively $T=T_{i n i}+i^{*} \Delta T$, where $i$ represents the number of iterations, $\Delta T$ is the temperature step of each iteration, and finally $I\left(n_{\text {wavenumber }}, T\right) \approx I_{90}\left(n_{\text {wavenumber }}\right)$, thus obtaining the curve $T\left(n_{\text {wavenumber }}\right)$.

Using the curve of temperature versus wave number, combined with the viewing angle, using the analytical model of the statistical band, the infrared spectral intensity at each viewing angle is calculated.

From the curve of temperature versus wave number, combined with the viewing angle $\theta$, substitute the following formula:

$$
\begin{aligned}
& I_{\theta}\left(n_{\text {wavenumber }}, T\left(n_{\text {wavenumber }}\right)\right)=\sigma T\left(n_{\text {wavenumber }}\right)^{4}\left(2 R_{\text {plume }}\right) L_{\text {plume }}\left(1-e^{-W / d}\right) / \pi
\end{aligned}
$$

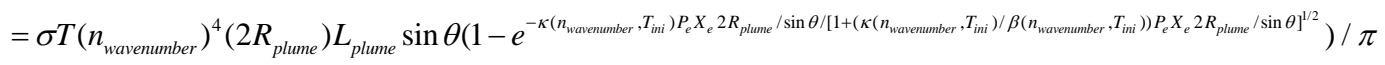

The infrared spectrum radiation curve of the exhaust plume corresponding to each viewing angle can be obtained. 


\section{RESULT ANALYSIS}

\subsection{Simulation of Radiation Intensity of Plume Spectral with Angle of View}

Figure 1 shows the comparison of the infrared spectroscopy intensity and the fine simulation of the exhaust plume with the change of the viewing angle. In this paper, CFD method 、line-by-line integration method and LOS method are used to calculate the infrared radiation characteristics of a rocket engine at a height of $20 \mathrm{~km}$. As a reference data, a fast generation method with the viewing angle is calculated and verified. In the figure, Cal represents the spectral radiance intensity calculated by fitting in this paper, and Exp represents the spectral radiance intensity obtained by the simulation of the fine modeling method. It can be seen from the figure that the emission peaks of $\mathrm{H}_{2} \mathrm{O}, \mathrm{CO}_{2}$ and $\mathrm{CO}$ are calculated, including $2.7 \mu \mathrm{m}, 4.3 \mu \mathrm{m}$ and $4.7 \mu \mathrm{m}$ spectra, and the overall fitted spectral radiation curve structure is consistent with the fine simulation. As the viewing angle decreases, the corresponding spectral radiation intensity also decreases, that is, the infrared radiation intensity of the positive lateral plume is the largest, which is consistent with the previous scholars' research conclusions. The spectral radiation intensity corresponding to the viewing angle of 80 degrees is quite good with the fine simulation matching. When the viewing angle is 40 degrees, the maximum error of the spectrum does not exceed $35 \%$. However, it should be noted that in the forward and backward calculations, the corresponding $\sin \theta=0$, that is, the forward and backward infrared radiation intensity estimation of the exhaust plume is not applicable in this paper. At the same time, in the forward and backward directions of the small angle, there is the occlusion of the projectile to make the estimated spectral radiation intensity deviate. Through repeated calculations, it is recommended that the estimated angular range is $10^{\circ} \leq \theta \leq 170^{\circ}$.

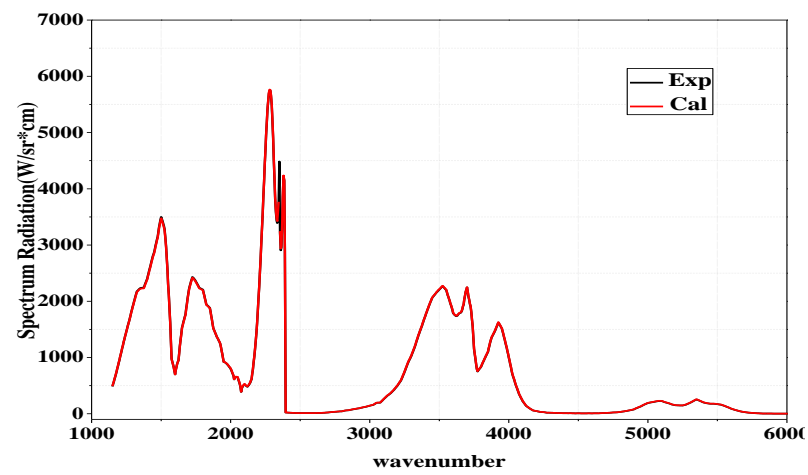

(a) $\operatorname{LOS}=80$ degree

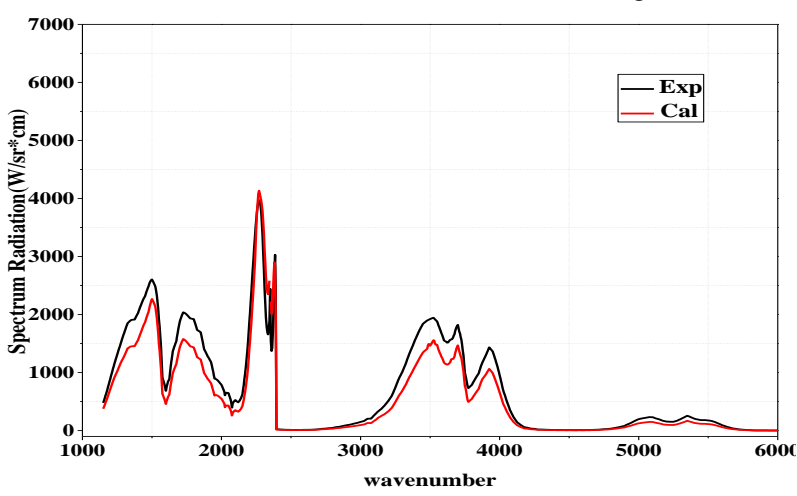

(c) $\operatorname{LOS}=40$ degree

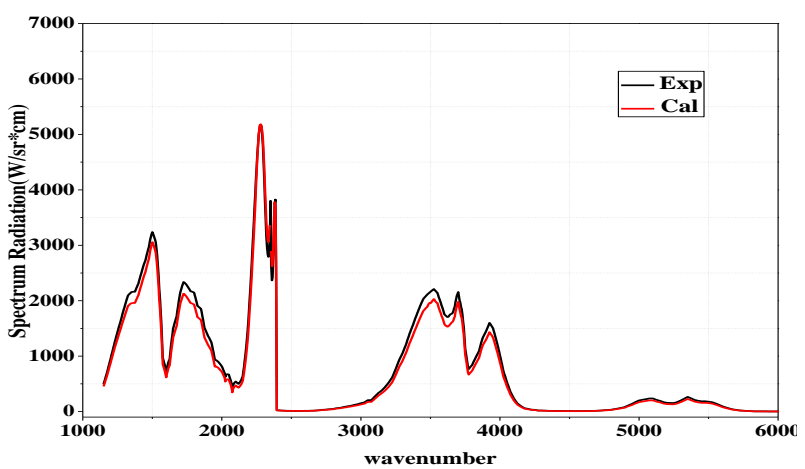

(b) $\operatorname{LOS}=60$ degree

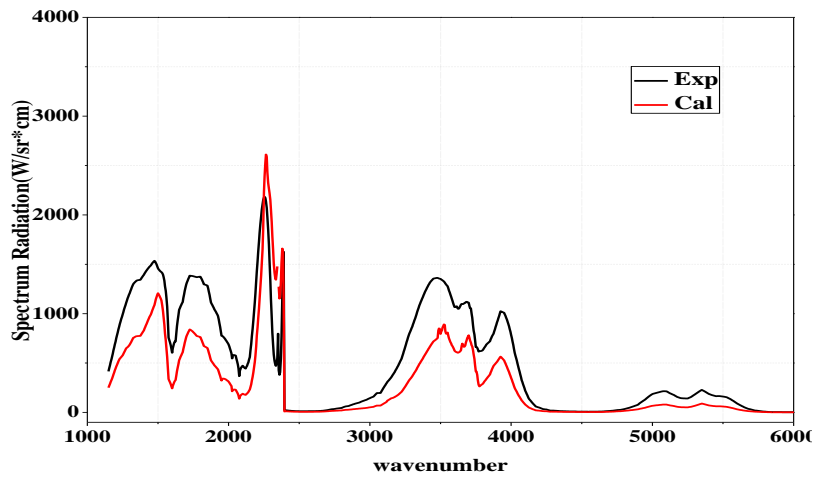

(d) $\operatorname{LOS}=20$ degree

Fig.1 Comparison of spectral intensity varying with viewing angle by the present method and the fine simulation 


\subsection{Fast calculation and Analysis of 3D Radiation field of Exhaust Plume}

Figure 2 is a comparison of the infrared radiation intensity and fine simulation of the exhaust plume as a function of the viewing angle. The radiation intensity of the plume at positive lateral is used as the reference, and the dimensionless is made. The figure shows the integrated radiation intensity of the typical emission band of $4.3 \mu \mathrm{m}$ and the measured contrast. It can be seen from the figure that the fitted radiation intensity is consistent with the fine angle simulation with the change of the viewing angle, and the positive lateral direction is the largest, and the forward and backward directions are the smallest. In the range of 90 to 50 viewing angles, the maximum error does not exceed 5\%. The maximum error is about $30 \%$ after the forward and backward small angles, indicating that the method can be quickly and accurately estimated.

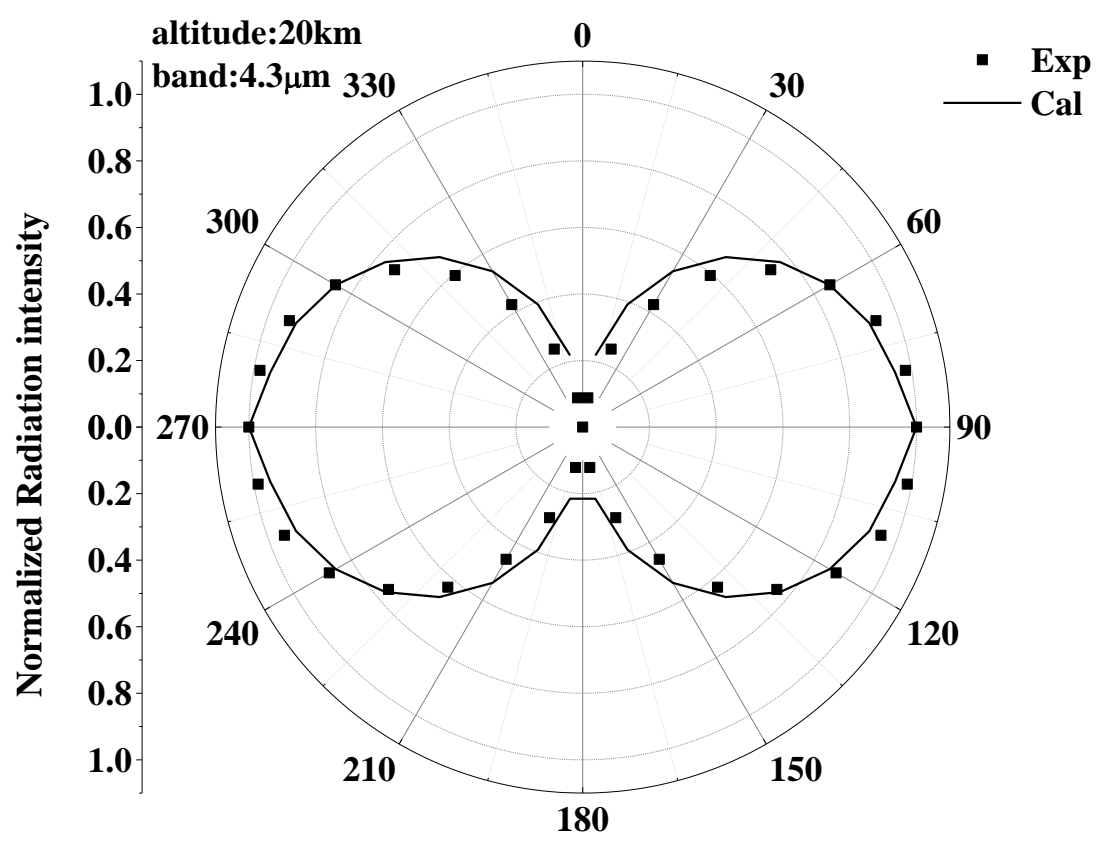

Fig.2 Comparison of the radiation intensity and fine simulation of the plume as a function of the viewing angle

Figure 3 shows the infrared radiation intensity field of the three-dimensional full-space exhaust plume generated by the method in this paper. The radiation intensity of the plume at positive lateral is used as the reference, and the dimensionless is made. It can be seen from the figure that due to the symmetry of the gas flow field of the exhaust plume, the infrared radiation intensity field of the exhaust plume also has spatial symmetry, which is characterized by a change with azimuth angle and constant with a change of the pitch angle. In terms of strength, the positive side is the strongest, and the forward and backward of the exhaust plume have the weakest infrared radiation intensity. 


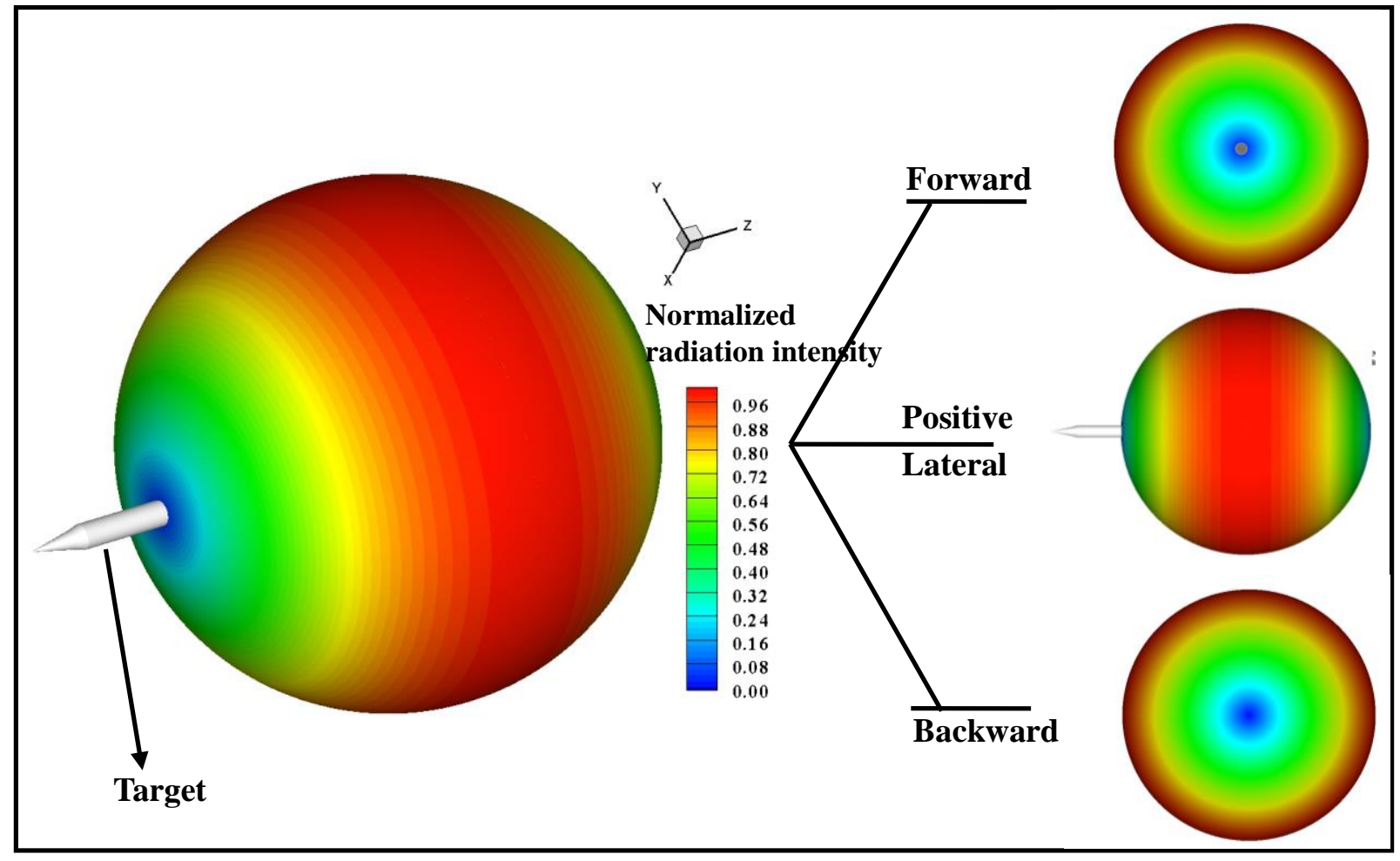

Fig.3 Three-dimensional full-space infrared radiation intensity field of the exhaust plume

\section{CONCLUSIONS}

In this paper, a method for rapidly generating the infrared spectrum of the plume with the viewing angle is established. The infrared radiation characteristics of the plume with the change of the viewing angle are studied. The variation of the plume with the viewing angle is pointed out. The positive lateral direction is the strongest, and the forward and backward directions are the weakest. On this basis, the three-dimensional spatial field of plume infrared radiation intensity is rapidly generated, which can visually display the spatial distribution law of plume intensity. A new efficient algorithm is provided to support the engineering application research of plume radiation characteristics.

\section{REFERENCES}

1. Sutton G P. Rocket propulsion elements, Wiley, New York, 1992, pp.549-563.

2. Raghunathan S, Benard E, Harrison R, et al. Plume Interference Effects on Missile Bodies[J]. Journal of Spacecraft \& Rockets, 2003, 40(40):136-138.

3. Simmons F S. Rocket Exhaust Plume Phenomenology [M]. California: The Aerospace Press, 2000.

4. Freeman G N, Ludwig C B, Malkmus W, et al. Development and Validation of Standardized Infrared Radiation Model (SIRRM). Gas/Particle Radiative Transfer Model[R]. AFRPL-79-55,1979. 
5. Bao Xingdong, Mao Hongxia, Wu Jie, Dong Yanbing. Research on Chemically Reacting Fluid Field and Infrared Radiation Signatures of a Rocket Motor Exhaust Plume[C]. The $4^{\text {th }}$ new Photoelectric Detection Technology and Application Symposium. Nanjing. October 2017.

6. Chai J C, Lee H S, Patankar S V. Finite Volume Method for Radiative Heat Transfer[J]. Journal of Thermophysics and Heat Transfer, 1994,8(3):419-425.

7. Howell J R, Perlmutter M. Monte Carlo Solution of Thermal Transfer though Radiant Media Between Gray Walls[J]. Journal of Heat Transfer-Transactions of the ASME,1964,86(1):116-122.

8. Selcuk N, Siddall G R. Two-Flux Spherical Harmonic Modeling of Two-Dimensional Radiative Transfer in Furnaces[J]. Journal of Heat and Mass Transfer, 1976,19:313-321.

9. Tan Heping, Xia Xinlin. Numerical computation of infrared radiation characteristics and transmission computational radiation[M]. Harbin: Harbin Institute of Technology Press, 2006.(in Chinese) 